\title{
Interactive comment on "Development of a high-resolution emission inventory and its evaluation through air quality modeling for Jiangsu Province, China” by Yaduan Zhou et al.
}

\section{Anonymous Referee \#2}

Received and published: 11 October 2016

General comments: This manuscript presents the development and evaluation of detailed emission inventories for major anthropogenic emission sources in Jiangsu Province, China. Emission is one of the major sources of uncertainty chemical transport modeling in China, where air pollution poses increasing threat to public health. Although it focuses only on one province (a relatively small emitter), which limits its scientific value to the broad ACP readership, this work could help establish procedures to develop and improve similar emission inventories in other provinces in this region. This manuscript could be improved in several key aspects to meet the requirements ACP. First, the presentation, in the current form, needs to be polished. There are several places in the text that the expression is either ambiguous or confusing. Second, 
the manuscript lacks detailed information on how these inventories were compiled (formula, parameters, etc), and where to find the key information to verify or reproduce the results reported here. Third, some of the materials included here are not relevant to the main theme. For example, the sensitivity of ozone and PM2.5 to 50\% reduction of NOx and VOC emissions has nothing to do with development and evaluation of the emission inventories. The authors may consider either broadening the theme (development, evaluation, and application of the Els) or removing the sensitivity section. Finally, the interpretation of some results need to be toned down or adjusted. Detailed comments and suggested changes are provided below.

Specific comments:

\section{L6: process(es).}

L26-30: Please reword this sentence. Define "unfavorable meteorology". Is the meteorology simulated by WRF inaccurate or the meteorology condition not conducive to pollution formation and accumulation? If the meteorology simulation is problematic, emission may reduce the model bias by offsetting opposite bias caused by meteorology inputs, but that does not warrant the quality of emission data per se.

Section 2.1: There are at least eight sectors considered here. Is Eq. (1) applied to all sectors or only to point sources? In the case that other formulas are used, please explain in more details (only citations are briefly provided here). It will be very useful to provide a table that includes the following key information: 1) sector; 2) sources included in that sector; 3) method to estimate emission; 4) key parameters and data sources to obtain the information; 5) special adjustment made.

L177: which model requirement?

L181-185: Can not understand this sentence. Please rephrase it. It seems a subtraction is involved here, by using data from different sources. What is the implication for uncertainty by subtracting data from different sources? 
Section 2.3. Again, a list of emission factors for each sector/source will be very useful. Maybe a table in the supplementary information.

L227: "by with"? Please revise the sentence.

L233-240. Please provide information of the spatial maps used to distribute emissions for each sector. Currently only the data for open burning have been given.

Interactive

comment

L280: "initial (concentration) and boundary conditions"

L290-291: Please elaborate how the vertical distribution is determined here. Is this applied to all sectors or just point sources?

L447-449: Is the agricultural GDP increase due to change in market price or commodity quantity?

Section 2 may need a sub-section to describe the measurement data (satellite and ground observations) used in this study.

Section 4.1 Using satellite NO2 data for emission evaluation has been well explored (check the literature for more details). There are several issues related to the method used here. Note OMI observes VCD, not emission. Therefore it may make more sense to compare the VCD from OMI to the equivalent from CMAQ, not the emission distribution directly, unless the nonlinear relationship between emission and VCD is accounted for. In addition the noise to signal ratio in OMI NO2 increases with decreasing VCD. The interpretation of the correlation between emission and OMI VCD needs to consider these factors.

L549-550: See the comments above.

Section 4.3: It is good to see that the new emission inventories can reduce high bias during extreme events when the meteorology is not correctly simulated. Do we have any results showing that this emission data can be used to improve prediction of real pollution events? This manuscript discusses the new emission data for the 
entire province. Why is only the model prediction over Nanjing discussed in the modelobservation comparison?

Section 5. As mentioned earlier, this section may not be necessary to support the main argument here. The interpretation of the results needs to acknowledge several caveats in the design and scope of these simulations. For instance, the brute force method does not consider nonlinearity in ozone response to precursor change. Large uncertainties exist in the emission dataset, including VOCs that have not been evaluated here, which will affect the chemistry and directionality of the ozone response. In addition, the sensitivity examines ozone response, but NOx and VOCs changes also result in changes in other pollutants, such as PM2.5. It is hence premature to draw such conclusion as in L770-772.

Interactive comment on Atmos. Chem. Phys. Discuss., doi:10.5194/acp-2016-567, 2016. 